\title{
Interference Management in OFDMA Femtocell Networks: Issues and Approaches
}

\author{
Nazmus Saquib, Ekram Hossain, Long Bao Le, and Dong In Kim
}

\begin{abstract}
One of the effective techniques of improving the coverage and enhancing the capacity and data rate in cellular wireless networks is to reduce the cell size (i.e., cell splitting) and transmission distances. Therefore, the concept of deploying femtocells over macrocell has recently attracted growing interests in academia, industry, and standardization forums. Various technical challenges towards mass deployment of femtocells have been addressed in recent literature. Interference mitigation between neighboring femtocells and between the femtocell and macrocell is considered to be one of the major challenges in femtocell networks because femtocells share the same licensed frequency spectrum with macrocell. Further, the conventional radio resource management techniques for hierarchical cellular system is not suitable for femtocell networks since the position of the femtocells is random depending on the users' service requirement. In this article, we provide a survey on the different state-of-the-art approaches for interference and resource management in orthogonal frequency-division multiple access (OFDMA)-based femtocell networks. A qualitative comparison among the different approaches is provided. To this end, open challenges in designing interference management schemes for OFDMA femtocell networks are discussed.
\end{abstract}

Index Terms-Femtocell, macrocell, OFDMA, interference management, LTE-Advanced systems, WiMAX.

\section{INTRODUCTION}

One of the major challenges for next generation wireless communication systems is to improve the indoor coverage and provide high-data-rate services to the users in a cost-effective manner and at the same time, to enhance network capacity. One of the traditional approaches of solving this problem is to make the transmitters and receivers closer to each other. However, this approach may not be economically feasible since it involves deploying more base stations (BSs) within the network. In this regard, home base stations, commonly known as femtocells, are considered as a promising option for the mobile operators to improve the network coverage, especially in the interiors of houses and buildings and to provide ubiquitous high speed connectivity to the end users or User Equipments (UEs). Femtocells or Femto Access Points (FAPs) are small, short-ranged $(10 \sim 30 \mathrm{~m})$ low powered $(10 \sim 100 \mathrm{~mW})$ access points developed to provide cost-effective and high-bandwidth services in next generation wireless communication systems. Femtocells operate in licensed spectrum owned by the mobile operator and enable Fixed Mobile Convergence (FMC) service by connecting to the cellular network via broadband communications links (e.g., DSL) [1].

One of the main advantages of femtocell deployment is the improvement of indoor coverage where macrocell base station (referred to as MeNB) signal is weak. Femtocells provide high data rate and improved quality-of-service (QoS) to the subscribers. It also lengthens the battery life of the mobile phones since the mobile phones do not need to communicate with a distant macrocell base station. Femtocells can easily be deployed by the end users in indoor environments on a "plug-and-play" basis. It saves the backhaul cost for the mobile operators since femtocell traffic is carried over wired residential broadband connections and reduces the traffic intensity at the macrocell network. Finally, femtocells can also be considered as an option towards the convergence of landline and mobile services. A recent study conducted by a market research company Informa Telecoms \& Media estimates that by 2014,114 million mobile users will be accessing mobile networks through femtocells [2]. This signifies that in the upcoming years femtocells could be an integral part of the next generation wireless communication systems.

In recent years, different types of femtocells have been designed and developed based on various air interface technologies, services, standards, and access control strategies. For example, $3 \mathrm{G}$ femtocells use Wideband Code-Division Multiple Access (WCDMA)-based air interface of Universal Mobile Telecommunication system (UMTS), which is also known as UMTS Terrestrial Radio Access (UTRA). The 3rd Generation Partnership Project (3GPP) refers to these 3G femtocells as Home Node Bs (HNBs). On the other hand, WiMAX (Worldwide Interoperability for Microwave Access) and Long Term Evolution (LTE) femtocells use Orthogonal FrequencyDivision Multiple Access (OFDMA). The LTE femtocells are referred to as Home evolved Node Bs (HeNBs).

In general, femtocells are designed to operate in one of three different access modes, i.e., closed access mode, open access mode, and hybrid access mode [3]. In closed access mode, a set of registered UEs belonging to Closed Subscriber Group (CGS) are allowed to access a femtocell. This type of femtocell access control strategy is usually applicable in residential deployment scenarios. However, in public places such as airports and shopping malls, open access mode of femtocells can also be used where any UE can access the femtocell and benefit from its services. This access mode is usually used to improve indoor coverage. In hybrid access mode, any UE may access the femtocell but preference would be given to those UEs which subscribe to the femtocell. In small business or enterprise deployment scenarios hybrid access mode of femtocells may be used [3].

\section{Technical Challenges in Femtocell DEPLOYMENT}

The mass deployment of femtocells gives rise to several technical challenges. One of the major challenges is in- 
terference management between neighboring femtocells and between femtocell and macrocell. In general, two types of interferences that occur in a two-tier femtocell network architecture (i.e., a central macrocell is underlaid/overlaid with 3G/OFDMA femtocells, respectively) are as follows:

- Co-tier interference: This type of interference occurs among network elements that belong to the same tier in the network. In case of a femtocell network, co-tier interference occurs between neighboring femtocells. For example, a femtocell UE (aggressor) causes uplink cotier interference to the neighboring femtocell base stations (victims). On the other hand, a femtocell base station acts as a source of downlink co-tier interference to the neighboring femtocell UEs. However, in OFDMA systems, the co-tier uplink or downlink interference occurs only when the aggressor (or the source of interference) and the victim use the same sub-channels. Therefore, efficient allocation of sub-channels is required in OFDMA-based femtocell networks to mitigate co-tier interference.

- Cross-tier interference: This type of interference occurs among network elements that belong to the different tiers of the network, i.e., interference between femtocells and macrocells. For example, femtocell UEs and macrocell UEs (also referred to as MUEs) act as a source of uplink cross-tier interference to the serving macrocell base station and the nearby femtocells, respectively. On the other hand, the serving macrocell base station and femtocells cause downlink cross-tier interference to the femtocell UEs and nearby macrocell UEs, respectively. Again, in OFDMA-based femtocell networks, cross-tier uplink or downlink interference occurs only when the same sub-channels are used by the aggressor and the victim.

Femtocells are deployed over the existing macrocell network and share the same frequency spectrum with macrocells. Due to spectral scarcity, the femtocells and macrocells have to reuse the total allocated frequency band partially or totally which leads to cross-tier or co-channel interference. At the same time, in order to guarantee the required QoS to the macrocell users, femtocells should occupy as little bandwidth as possible that leads to co-tier interference. As a result, the throughput of the network would decrease substantially due to such co-tier and cross-tier interference. In addition, severe interference may lead to "Deadzones", i.e., areas where the QoS degrades significantly. Deadzones are created due to asymmetric level of transmission power within the network and the distance between macrocell UE and macrocell base station. For example, a macrocell UE located at a cell edge and transmitting at a high power will create a deadzone to the nearby femtocell uplink transmission due to co-channel interference. On the other hand, in the downlink transmission, due to high path-loss and shadowing effect, a cell edge macrocell UE may experience severe co-channel interference from the nearby femtocells. Thus, it is essential to adopt an effective and robust interference management scheme that would mitigate the co-tier interference and reduce the cross-tier interference considerably in order to enhance the throughput of the overall network.

In OFDMA-based femtocell networks, due to the flexibility in spectrum allocation, orthogonal sub-carriers can be assigned to femtocells and macrocells. This gives OFDMA-based femtocells an edge over CDMA systems in terms of utilizing the frequency spectrum resources efficiently. Fig. 1 illustrates all possible interference scenarios in an OFDMA-based femtocell network. If an effective interference management scheme can be adopted, then the co-tier interference can be mitigated and the cross-tier interference can be reduced which would enhance the throughput of the overall network.

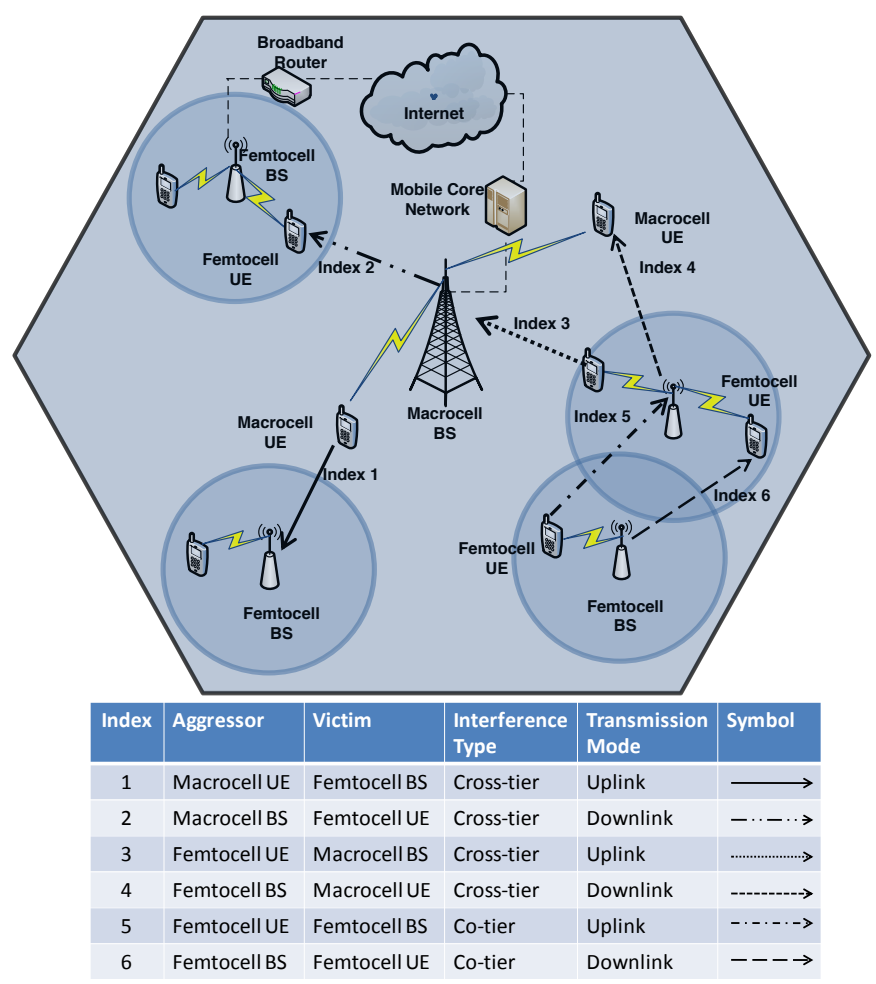

Fig. 1. Interference scenarios in OFDMA-based femtocell networks.

Other challenges in femtocell deployments include: handoff and mobility management, timing and synchronization, auto-configuration, and security. An effective and efficient mobility management and handover scheme (macrocell-tofemtocell, femtocell-to-macrocell and femtocell-to-femtocell) is necessary for mass deployment of femtocells in UMTS and LTE networks. The scheme should have low complexity and signaling cost, deal with different access modes and perform proper resource management beforehand for efficient handover. Timing and synchronization is one of the major challenges for femtocells since synchronization over IP backhaul is difficult, and inconsistent delays may occur due to varying traffic congestion. Since the femtocells are required to operate on a "plug-and-play" basis, it is important that femtocells can organize and configure autonomously and access the radio network intelligently so that they only cause minimal impact on the existing macrocell network. Since femtocells could be vulnerable to malicious attacks (e.g., masquerading, eavesdropping, man-in-the-middle attack etc.), 
enhanced authentication and key agreement mechanisms are required to secure femtocell networks.

In this article, we give an overview of the different interference management techniques for OFDMA-based femtocell networks presented in the recent literature. To this end, we will provide a qualitative comparison among these techniques based on some important criteria. We will conclude the article outlining some open challenges related to interference management in OFDMA-based femtocell networks.

\section{Interference MANAGEMENT APPROACHES}

Different techniques such as cooperation among macrocell BSs (i.e., MeNBs) and femtocell BSs (i.e., HeNBs), formation of groups of HeNBs and exchange of information (such as path loss, geographical location, etc.) among neighboring HeNBs, accessing the spectrum intelligently, etc. can be considered to reduce co-tier and cross-tier interferences. In the following, we provide an overview of the different approaches for interference mitigation in two-tier OFDMA femtocell networks. These approaches consider uplink and/or downlink transmissions as well as co-tier and/or cross-tier interference.

\section{A. Femto-aware spectrum arrangement scheme}

In [4], Yi Wu et al. propose a femto-aware spectrum arrangement scheme to avoid uplink cross-tier interference between a macrocell and femtocells. In this scheme, the allocated frequency spectrum for any macrocell coverage area is divided into two parts: the macrocell dedicated spectrum part and macrocell-femtocell shared spectrum part. It is assumed that shared spectrum allocated to femtocells (i.e., HeNBs) is configured by the mobile operator. Thus, the macrocell base station (i.e., MeNB) has adequate knowledge of the shared frequency spectrum. Based on this knowledge, the MeNB develops an interference pool which includes the macrocell UEs that pose a threat to the nearby HeNBs. These macrocell UEs are thus assigned a portion of the spectrum dedicated for macrocell usage which reduces/mitigates the uplink cross-tier interference and solves the uplink deadzone problem.

Fig. 2 illustrates the femto-aware spectrum arrangement scheme, where macrocell UE4, macrocell UE5, and macrocell UE6 pose potential threat of cross-tier interference on their prospective nearby HeNBs. Therefore, these macrocell UEs are put into the femtocell-interference pool by the MeNB and are assigned a dedicated portion of the total frequency spectrum in order to mitigate co-channel interference. On the other hand, since other macrocell UEs (i.e., macrocell UE1, macrocell UE2, and macrocell UE3) are not close to any $\mathrm{HeNB}$, they share the rest of the frequency spectrum along with the femtocell UEs (i.e., femtocell UE1, femtocell UE2, and femtocell UE3). However, this scheme does not consider inter-HeNB interference and may be inefficient if the number of macrocell UEs near the HeNB increases.

\section{B. Clustering of femtocells}

In [5], a framework is presented to reduce downlink interference (both cross-tier and co-tier) and enhance the spectral

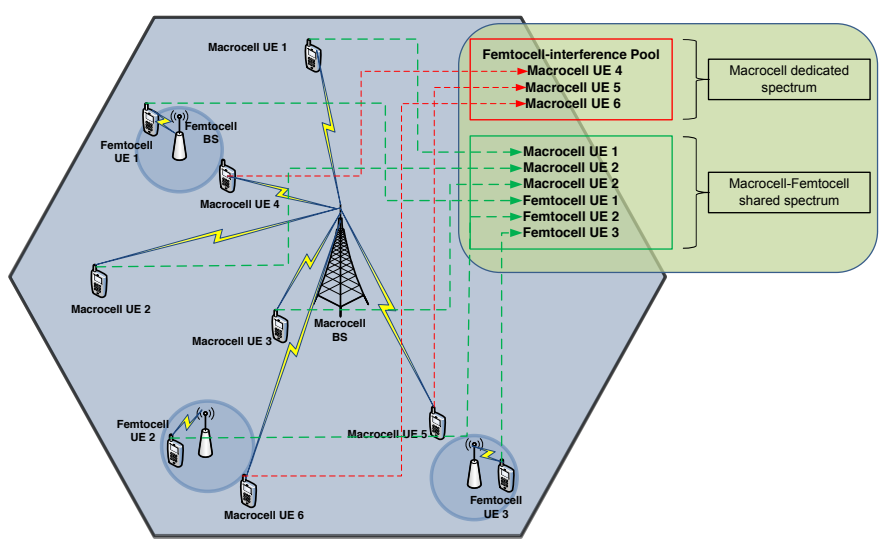

Fig. 2. Femto-aware spectrum arrangement scheme.

efficiency for an OFDMA-based closed access femtocell network. In this framework, a Femtocell System Controller (FSC) per macrocell obtains all the necessary knowledge of HeNB system configuration (i.e., position information of HeNBs and macrocell UEs) and performs the necessary computations. To mitigate interference, the scheme encompasses a combination of dynamic frequency band allocation among HeNBs and $\mathrm{MeNB}$, and clustering of HeNBs based on their geographical locations. In this scheme, a portion of the entire frequency band is dedicated to the MeNB users and the rest is reused by the MeNB and HeNBs. The advantage of allocating a portion of the frequency band strictly for MeNB users is that it can solve the MeNB UE downlink deadzone problem and guarantee users' QoS requirement. However, the portion of the frequency band, which is shared, is determined by the total number of HeNB clusters obtained through a clustering algorithm. The clustering algorithm allocates HeNBs into different frequency reuse clusters and UEs of different HeNBs in the same cluster use the same sub-channels allocated from the shared frequency band. Based on the geographical locations of the HeNBs, the threshold distance for clustering interference is calculated. If the Euclidean distance between any two HeNBs is less than the threshold distance, then they are assigned to different clusters to avoid co-tier and crosstier interferences. Simulation results show that high spectrum efficiency is achieved as the probability of cross-tier spectrum reuse becomes higher than $97.4 \%$. This signifies that the problem regarding macrocell UE downlink deadzone around HeNBs is effectively solved (e.g., the probability of one macrocell UE lying in the deadzone is below 2.4\%). For the proposed scheme, simulation results also show a significant improvement of the femtocell user capacity (at most 200 HeNBs per macrocell coverage area).

In [6], an energy-efficient interference mitigation scheme is presented for closed access HeNBs grouped in a neighborhood area based on their geographical locations. In this scheme, inter-femtocell or co-tier interference among neighboring $\mathrm{HeNBs}$ is minimized by reducing the unnecessary Available Intervals (AI) in Low Duty Operation (LDO) mode for HeNBs. According to the IEEE $802.16 \mathrm{~m}$ standard, a HeNB in the operation state may enter the LDO mode if no UE 
exists in its coverage zone, or if all UEs in the coverage are in sleep/idle mode. In the LDO mode, a HeNB switches alternately between available interval (AI) and unavailable interval (UAI) modes. During UAI, a HeNB becomes inactive on the air interface. During AI, the HeNB may become active on the air interface by transmitting preambles to the new incoming UE for synchronization purposes. However, the HeNB in the LDO mode still has AIs even though there is no UE that will access the HeNB in near future. These unnecessary AIs cause co-tier interference for CGS HeNBs.

In the proposed scheme, unnecessary AIs are decreased which results in reduction of co-tier interference among neighboring HeNBs. The main idea behind reducing such interference is to cluster/group the neighboring HeNBs based on their geographical locations. In each cluster, one HeNB is designated as the leader and the adjacent HeNBs are referred to as members. According to the IEEE $802.16 \mathrm{~m}$ standard, a newly installed HeNB scans the surrounding area to search for neighbor HeNBs in its initialization state. Since it is assumed that the network has global knowledge about the topology of the network, the scanning report may include the group configuration in the network (i.e., the leader and the members of the group based on the HeNB ID). If a newly installed HeNB receives the preamble signal from the leader above a defined threshold then it becomes a member of the group, otherwise, it will form a new group and assign itself as the leader of the group. The leader requires having AIs in its LDC pattern so that the arrival of a UE at the group can discover the existence of the group by detecting the leader, even though the members in the group stay in UAI. As soon as the leader senses the arrival of the UE, it sends a message to the target HeNB to activate its AI in the LDC pattern so that the UE can detect the target HeNB and connect to it. In this pattern, the unnecessary AI in the LDO mode of HeNB is reduced resulting in power conservation of $\mathrm{HeNB}$ and at the same time the co-tier interference is minimized. Through analysis and simulation it is shown that for the proposed scheme, the gain in terms of co-tier interference reduction time and energy saving is up to $90 \%$ in comparison with conventional LDO scheme in the IEEE $802.16 \mathrm{~m}$ standard.

\section{Beam subset selection strategy}

The authors in [7] propose an orthogonal random beamforming-based cross-tier interference reduction scheme in closed-access two-tier femtocell networks. The macrocell beam subset selection strategy is based on the number of macrocell UEs and the intensity of HeNBs in the network. The MeNB selects the beam subset and the users for each channel based on the signal-to-interference-plus-noise (SINR) information for all the channels which is fedback by the macrocell UEs. The main objective is to enhance the throughput of the network by optimizing the trade-off between multiplexing gain and multiuser interference (cross-tier) based on adaptive selection of optimal number of beams using max-throughput scheduler at the MeNB. The adaptive selection of the number of beams decreases cross-tier interference, and provides spatial opportunity to HeNBs to access the spectrum in an opportunistic manner. In addition, distributed power control mechanism for HeNBs integrated with the proposed scheme reduces cross-tier interference significantly.

\section{Collaborative frequency scheduling}

Co-channel uplink and downlink cross-tier interference can be mitigated if a HeNB can avoid using the macrocell resource blocks that belong to its nearby macrocell UEs through efficient spectrum sensing. However, the spectrum sensing results for HeNB may be impaired due to misdetection, false alarm, and improper timing synchronization. To deal with this problem, a framework for OFDMA-based HeNBs is provided in [8] where the scheduling information for macrocell UEs' (both uplink and downlink) is obtained from the MeNB through backhaul or air interface. This information is used to improve the spectrum sensing results for HeNB and to utilize the resource blocks associated with far-away macrocell UE in the uplink and downlink transmission. The key features of the proposed framework are as follows:

- HeNB receives the macrocell UEs scheduling information for uplink and downlink from the MeNB.

- HeNB performs spectrum sensing for finding the occupied parts of the spectrum. The occupied parts of the uplink spectrum can be determined through energy detection.

- HeNB compares the spectrum sensing results with the obtained scheduling information to decide about the spectrum opportunities.

Since the HeNB accesses the spectrum in an opportunistic manner, the authors analyze the impact of Inter-carrier Interference (ICI) from macrocell UEs to femtocell which is severe in the uplink transmission. The ICI is basically due to the asynchronous arrival of macrocell UE signals at the femtocell. Through simulation (using Okumura-Hata model of radio propagation) it is shown that the variation of the ICI power depends on center frequency, height of the femtocell, and the size of the Cyclic Prefix (CP). A lower center frequency and a higher femtocell height increase the received ICI power at HeNB. In addition, if the macrocell UEs' signal arrival time at HeNB exceeds the CP duration then the orthogonality between the sub-carriers is disrupted leading to ICI. Also, different subcarrier assignment schemes result in different ICI.

\section{E. Power control approach}

Power control methods for cross-tier interference mitigation generally focus on reducing transmission power of HeNBs. These methods are advantageous in that the MeNB and HeNBs can use the entire bandwidth with interference coordination. Dynamic or adjustable power setting, which is preferred over fixed HeNB power setting, can be performed either in proactive or in reactive manner each of which again can be performed either in open loop power setting (OLPS) or closedloop power setting (CLPS) mode. In the OLPS mode, the HeNB adjusts its transmission power based on its measurement results or predetermined system parameters (i.e., in a proactive manner). In the CLPS mode, the HeNB adjusts its 
transmission power based on the coordination with MeNB (i.e., in a reactive manner). Also, a hybrid mode can be used where the HeNB switches between the two modes according to the operation scenarios [9]. Another related concept is power control for HeNBs on a cluster basis in which the initial power setting for the HeNBs is done opportunistically based on the number of active femtocells in a cluster (Fig. 3) [10]. For this, centralized sensing can be used by which an MeNB can estimate the number of active femto cells per cluster and broadcast the interference allowance information to femtocells for their initial power setting. Alternatively, distributed sensing can be used where each cell senses if the others are active in the same cluster and adjusts its initial power setting accordingly.

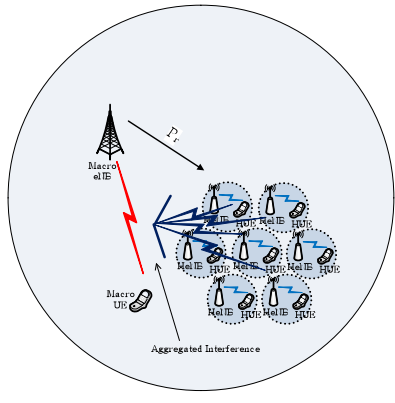

(a) Centralized sensing

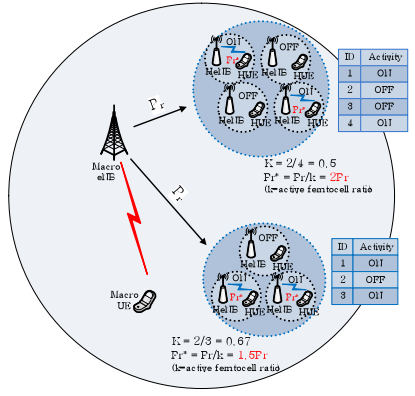

(b) Distributed sensing
Fig. 3. Sensing-based opportunistic power control (from [10]).

Game theoretic models can be used to design and analyze distributed power control methods in a heterogeneous cellular wireless network with macrocells and femtocells. Two broad categories of game theoretic models are noncooperative and cooperative game models. In [15], a distributed power control allocation problem is formulated for downlink transmission of OFDMA-based femtocells overlaid upon a macrocell network. The problem is modeled as a noncooperative game, namely, a Stackelberg game, where the throughput of each station in the network is maximized under power constraints. In this game, the macrocell UEs are referred as the leaders and the femtocell UEs are considered to be the followers. The game is divided into two sub-games: the sub-game comprised of the set of leaders, referred to as the upper sub-game, and the sub-game comprised of the set of followers, referred to as the lower sub-game. The players in each sub-game compete with each other in a non-cooperative manner to reach a sub-game Nash equilibrium, which is the solution of the power control game.

\section{F. Fractional Frequency Reuse (FFR) and resource partition- ing}

The basic mechanism of this method divides the entire frequency spectrum into several sub-bands. Afterwards, each sub-band is differently assigned to each macrocell or sub-area of the macrocell. Since the resource for MeNB and HeNB is not overlapped, interference between MeNB and HeNB can be mitigated. In [11], the authors propose a frequency sharing mechanism that uses frequency reuse coupled with pilot sensing to reduce cross-tier/co-channel interference between macrocell and femtocells. In this scheme, FFR of 3 or above is applied to the macrocell. When a HeNB is turned on, it senses the pilot signals from the MeNB and discards the subband with the largest received signal power, and thus uses the rest of the frequency sub-bands resulting in an increased SINR for macrocell UEs. The overall network throughput is enhanced by adopting high-order modulation schemes.

In [12], another interference management scheme for LTE femtocells is presented based on FFR. The scheme avoids downlink cross-tier interference by assigning sub-bands from the entire allocated frequency band to the HeNBs that are not being used in the macrocell sub-area. In the proposed scheme, the macrocell is divided into center zone (corresponding to $63 \%$ of the total macrocell coverage area) and edge region including three sectors per each region. The reuse factor of one is applied in the center zone, while the edge region adopts the reuse factor of three. The entire frequency band is divided into two parts and one of them is assigned to the center zone. The rest of the band is equally divided into parts and assigned in the three edge regions.

Fig. 4(b) illustrates the allocation of frequency sub-bands within the macrocell sub-areas. The sub-band $\mathrm{A}$ is used in the center zone $(\mathrm{Cl}, \mathrm{C} 2$, and $\mathrm{C} 3)$, and sub-bands $\mathrm{B}, \mathrm{C}$, and $\mathrm{D}$ are used in regions $X l, X 2$, and $X 3$, respectively. Now, when a HeNB is turned on, it senses the neighboring MeNB signals, compares the Received Signal Strength Indication (RSSI) values for the sub-bands, and chooses the sub-bands which are not used in the macrocell sub-area. In addtion, if the HeNB is located in the center zone then it excludes the sub-band that is used in the center zone as well as the one that is used by the mactocell in the edge region of the current sector. For example, if a HeNB is located in edge region $X 1$, then it would exclude sub-band $B$ which is used by macrocell UEs, and select sub-band A, C, or D. However, if a HeNB is located in center zone $\mathrm{Cl}$, then it avoids sub-band $\mathrm{A}$ and at the same time sub-band B since the RSSI for this sub-band is comparatively higher for that HeNB. In this way, this scheme mitigates co-tier and cross-tier interference. Simulation results show that, the scheme offers throughput gains of $27 \%$ and $47 \%$ on average, when compared with the FFR-3 scheme (with no center zone) and a scheme with no FFR, respectively.

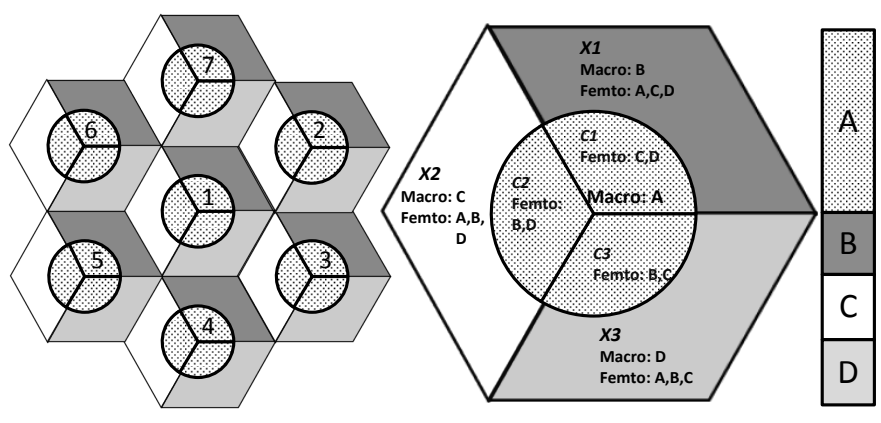

(a)

(b)

Fig. 4. Interference management scheme using FFR.

The two schemes described above use a fixed partitioning, which would cause a loss in throughput performance due 
to inefficient use of the bandwidth resources. A dynamic partitioning scheme (in both time and frequency domain) can be used for bandwidth sharing which minimizes cross-tier interference. In [13], an adaptive FFR scheme is presented to minimize downlink interference caused by the HeNBs in the vicinities of a macrocell. The proposed scheme adopts FFR radio resource hopping or orthogonal FFR radio resource allocation based on the density (e.g., high or low) and location information (e.g., inner region or outer region) of the HeNBs. The location information of the HeNBs may be obtained and maintained within the network through using registered physical address associated with the broadband IP (Internet Protocol) address that a HeNB uses. The proposed scheme only deals with the cross-tier interference posed by the HeNBs located (inner region) near the MeNB. If the HeNB is situated in a high dense inner region, then orthogonal sub-channels are adopted by the HeNBs. Otherwise, the HeNB selects a subchannel arbitrarily, utilizes it for a certain period of time, and then hops to other sub-channels. The proposed scheme reduces downlink cross-tier interference.

Note that resource partitioning method can be used along with power control (thus resulting in a hybrid approach) to reduce co-tier and cross-tier interferences.

\section{G. Cognitive approach}

Cognitive radio approach based on distributed spectrum sensing can be used for interference mitigation in femtocell networks. In [14], an efficient downlink co-tier interference management scheme for an OFDMA-based LTE system is proposed where the path-loss information is shared among HeNB neighbors. In addition, adjacent HeNBs share the information related to the usage of LTE Component Carriers (CC), achieved based on carrier aggregation technique leading to a sub-channel, in a distributed manner. The exchange of information between HeNBs may be done via femtocell gateway (HeNB GW) or over-the-air (OTA) method. The HeNB GW is considered to be an intermediate node between HeNBs and mobile core network that manages the inter-HeNB coordination messages via S1 connection. On the other hand, the OTA method includes a direct link between HeNB and MeNB.

In the proposed scheme, when a HeNB is turned on, it identifies the adjacent neighbors and obtains the knowledge of the CCs used by the neighbors. The main idea of the scheme is that, each HeNB estimates the co-tier interference based on the path-loss information, capitalizes the knowledge of the usage of CCs by the neighbors, and accesses the spectrum intelligently to minimize interference. The selection of $\mathrm{CC}$ is done in such a way that, each HeNB selects the $\mathrm{CC}$ which is not used by the neighbor or the $\mathrm{CC}$ that is occupied by the furthest neighbor or the $\mathrm{CC}$ that is occupied by the least number of neighbors (in a chronological order as mentioned). Simulation results show a significant reduction in co-tier interference and signaling overhead within the network when compared with another cognitive based HeNB co-tier interference management technique.

Fig. 5 illustrates a scenario of co-tier interference management (downlink) of HeNBs through cognitive approach. In this scenario, let us consider that the available CCs for HeNBs are $\mathrm{CC} 1, \mathrm{CC} 2, \mathrm{CC} 3$, and CC4. In Fig. 5(a), since HeNB1 and HeNB3 are adjacent to each other, they select different CCs. On the other hand, since HeNB2 is a neighbor of neither HeNB1 nor HeNB3, it selects any one pair of available CCs (e.g., CC1 and CC2). Now, under such femtocell deployment, when HeNB4 is turned on, it discovers its adjacent neighbors, i.e., HeNB1 and HeNB2. Through inter-HeNBs coordination mechanism, HeNB4 obtains the information related to the usage of CCs of its adjacent neighbors. Thus, in order to avoid co-tier interference, when HeNB4 selects CCs for the downlink transmission, it selects the CCs (i.e., CC3 and CC4) which are different from those used by from HeNB1 and HeNB2. Furthermore, in Fig. 5(b), when HeNB5 is turned on, it identifies the adjacent neighbors (i.e., HeNB1, HeNB3, and HeNB4), and obtains the knowledge of the CCs used by the neighbors. Under these circumstances, HeNB5 selects the CCs that are occupied by the furthest neighbor, i.e., HeNB1. To this end, HeNB5 selects $\mathrm{CC} 1$ and $\mathrm{CC} 2$ for downlink transmission to reduce co-tier interference.

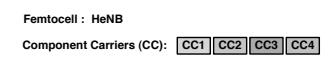

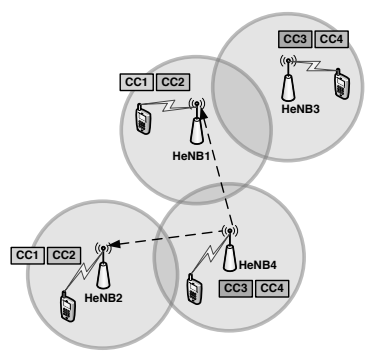

(a)

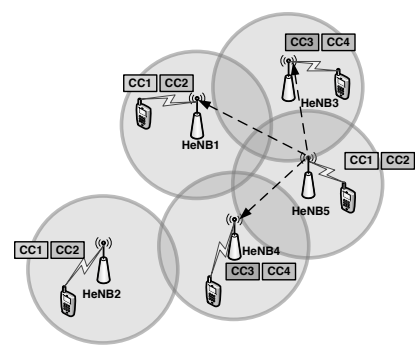

(b)
Fig. 5. Interference management through cognitive approach.

\section{Qualitative Comparison Among InTERFEREnCE MANAGEMENT APPROACHES}

Table I provides a qualitative comparison among different interference management schemes. The "efficiency" of a scheme depends on whether it (i) mitigates/significantly reduces both co-tier and cross-tier interferences; (ii) is applicable for both uplink and downlink transmissions; (iii) considers coordination among HeNBs and MeNB, or capitalizes on minimal amount of information, i.e., path-loss, geographical location, or usage of the spectrum or sub-band among nearby HeNBs and/or among HeNBs and MeNB; (iv) handles ICI (e.g., by using frequency scheduling or any other method); (v) adopts an adaptive power control mechanism; (vi) corresponds to opportunistic access of the spectrum by the HeNBs based on RSSI value from MeNB signals; (vii) reduces the unnecessary AIs of LDO mode for HeNBs; (viii) is scalable and robust, i.e., implementable for mass deployment of HeNBs; and (ix) is applicable for all 3 types of access modes (i.e., closed, open, and hybrid). If any scheme attains majority (more than 5) of these attributes, then we consider the efficiency of the 
scheme to be high. We consider the efficiency of a scheme to be moderate if it attains 3-5 of the aforementioned attributes.

For example, the efficiency of cognitive approach is considered to be moderate since it is capable of handling both cross-tier and co-tier interferences with minimal amount of information (i.e., information about usage of sub-bands) exchange among neighboring HeNBs, applicable for all types of access modes of HeNBs, and more importantly, it accesses the spectrum in an opportunistic manner causing minimal harm to the nearby macrocell UEs. The collaborative frequency scheduling scheme is considered to be highly efficient since it significantly reduces cross-tier and co-tier interferences for mass deployment of HeNBs in both uplink and downlink transmission, handles ICI problem, and allows the HeNBs to opportunistically access the spectrum based on only the scheduling information of macrocell UEs that is exchanged among HeNBs and MeNB.

The "complexity" of each scheme increases with (i) the amount of information exchanged between neighboring HeNBs, (ii) the amount of information exchanged between HeNBs and MeNB, (iii) formation of clusters among HeNBs, (iv) algorithm executed in the HeNBs and/or in the MeNB to allow the HeNBs to access the spectrum opportunistically etc. The more information exchanged among HeNBs or between HeNBs and MeNB, the more signaling overhead is introduced, and more processing is done in both HeNBs and MeNB, increasing the complexity of the scheme. For example, the complexity of the beam subset selection strategy scheme is considered to be high since it requires the channel state information from all macrocell UEs to determine the optimal number of beams every time along with extensive coordination between HeNBs and MeNB regarding the spectrum access (thus increasing the signaling overhead). Also, the HeNBs have to run iterative power control algorithm to minimize interference.

Selection of an interference management scheme depends on the desired trade-off between complexity and efficiency. We recommend to adopt FFR as an interference management scheme for two-tier femtocell networks since it requires minimal/no coordination among HeNBs and MeNB (and hence reduces the signaling overhead, and thus the complexity of the system), opportunistically accesses the spectrum based on only RSSI value from MeNB signals, and it effectively solves the problem of cross-tier and co-tier interferences in uplink and downlink transmission for difference access modes of HeNBs. Consequently, it can increase the throughput of the network by a large margin, and can be used when the average number of HeNBs per macrocell is very high (about 180-200) while maintaining the QoS requirements of macrocell UEs. Currently, FFR is being considered as an effective interference management scheme for OFDMA-based two-tier femtocell networks [2].

\section{Open Challenges}

To enable mass deployment of femtocells, it is essential to develop distributed interference management schemes which primarily satisfy the QoS requirements of macrocell and femtocell UEs and at the same time enhances the capacity and coverage of the network. Such schemes should incur low overhead for coordination among macrocell BSs (i.e., MeNBs), and also should be able to integrate mobility management with different access modes and synchronization issues while keeping the complexity as minimal as possible. The interference management solution would strongly depend on the employed radio access technology (e.g., CDMA or OFDMA) and access mode (i.e., closed, open, or hybrid). In particular, adaptive admission control, power control, and advanced communication strategies such as interference cancellation and beamforming for multiple-antenna transceivers are important techniques to mitigate co-tier and cross-tier interferences. For example, by using beamforming techniques femtocells can form antenna beams toward their UEs while nulling interference caused to macrocell UEs. In addition, macrocells would have higher priority in accessing the spectrum; therefore, suitable admission control mechanisms should be activated when femtocells create intolerable interference for macrocell UEs.

For OFDMA-based femtocell networks, if different sets of subchannels are assigned to macrocells and femtocells, crosstier interference can be completely eliminated. However, to improve the spectrum utilization, a more efficient spectrum assignment method can be adopted.

Also, hybrid interference management schemes which combine power control with resource partitioning are promising. Power control schemes are advantageous in that MeNB and HeNB can use the entire bandwidth with interference coordination for both control and data channels. However, for this, the HeNB measurement scheme for power setting would need to be standardized. Also, such a scheme may not be fully effective when a macro UE is located very close to a HeNB. With resource partitioning schemes, interference between MeNB and HeNB can be eliminated. However, multiple frequency bands are required. The merits of both the approaches can be exploited in a hybrid scheme, the design of which is not trivial.

\section{CONCLUSION}

The femtocell technology can provide many advantages to the mobile subscribers and the service providers. Thus, femtocells could be viewed as a promising option for next generation wireless communication networks such as OFDMAbased LTE-Advanced and WiMAX networks. We have provided a survey of different techniques to cope with the co-tier and cross-tier interference problem in OFDMA-based two-tier femtocell networks. With efficient interference management schemes, the network capacity and coverage can be increased that benefit both the subscribers and the operators.

\section{ACKNOWLEDGMENT}

This research was also supported in part by NSERC, Canada, and in part by the MKE (Ministry of Knowledge Economy), South Korea, under the ITRC (Information Technology Research Center) support program supervised by the NIPA (National IT Industry Promotion Agency (NIPA-2011(C1090-1111-0005)). 
TABLE I

QUALITATIVE COMPARISON AMONG DIFFERENT INTERFERENCE MANAGEMENT SCHEMES

\begin{tabular}{|c|c|c|c|c|c|}
\hline Scheme & $\begin{array}{c}\text { Transmission } \\
\text { mode }\end{array}$ & $\begin{array}{c}\text { Cooperation among } \\
\text { HeNBs and MeNB }\end{array}$ & $\begin{array}{c}\text { Access } \\
\text { mode }\end{array}$ & Complexity & Efficiency \\
interference
\end{tabular}

\section{REFERENCES}

[1] V. Chandrasekhar and J. G. Andrews, "Femtocell Networks: A Survey," IEEE Communications Magazine, vol. 46, no. 9, pp. 59-67, Sept. 2008.

[2] www.femtoforum.org

[3] A. Golaup, M. Mustapha, and L. B. Patanapongipibul, "Femtocell Access Control Strategy in UMTS and LTE," IEEE Communications Magazine, vol. 47, no. 9, pp. 117-123, Sept. 2009.

[4] W. Yi, Z. Dongmei, J. Hai, and W. Ye, "A Novel Spectrum Arrangement Scheme for Femtocell Deployment in LTE Macrocells," Proc. IEEE 20th Symposium on Personal, Indoor and Mobile Radio Communications, pp. 6-11, 13-16 Sept. 2009.

[5] H. Li, X. Xu, D. Hu, X. Qu, X. Tao, and P. Zhang,"Graph Method Based Clustering Strategy for Femtocell Interference Management and Spectrum Efficiency Improvement," Proc. IEEE 6th International Conference on Wireless Communications Networking and Mobile Computing (WiCOM), pp. 1-5, 23-25 Sept. 2010.

[6] H. Widiarti, S. Pyun, and D. Cho, "Interference Mitigation Based on Femtocells Grouping in Low Duty Operation," Proc. IEEE 72nd Vehicular Technology Conference Fall (VTC'10-Fall), pp. 1-5, 6-9 Sept. 2010.

[7] S. Park, W. Seo, Y. Kim, S. Lim, and D. Hong,"Beam Subset Selection Strategy for Interference Reduction in Two-tier Femtocell Networks," IEEE Transactions on Wireless Communications, vol. 9, no. 11, pp. 34403449, Nov. 2010

[8] M. E. Sahin, I. Guvenc, Moo-Ryong Jeong, H. Arslan, "Handling CCI and ICI in OFDMA Femtocell Networks through Frequency Scheduling," IEEE Transactions on Consumer Electronics, vol. 55, no. 4, pp. 19361944, Nov. 2009.

[9] 3GPP R1-105238, "Further Discussion on HeNB Downlink Power Setting in HetNet," 3GPP RAN1 Meeting, Xi?n, China, Oct. 2010.

[10] M. S. Jin, S. Chae, and D. I. Kim, "Per Cluster Based Opportunistic Power Control for Heterogeneous Networks," in Proc. IEEE VTC'11Spring, Budapest, Hungary, May 2011.

[11] T. Kim and T. Lee, "Throughput Enhancement of Macro and Femto Networks by Frequency Reuse and Pilot Sensing," Proc. IEEE International Performance, Computing and Communications Conference (IPCCC), pp. 390-394, Dec. 2008.

[12] L. Poongup, L. Taeyoung, J. Jangkeun, and S. Jitae, "Interference Management in LTE Femtocell Systems Using Fractional Frequency Reuse," Proc. 12th International Conference on Advanced Communication Technology, vol. 2, pp. 1047-1051, 7-10 Feb. 2010.

[13] R. Juang, P. Ting, H. Lin, and D. Lin, "Interference Management of Femtocell in Macro-cellular Networks," Proc. Wireless Telecommunications Symposium (WTS'10), pp.1-4, 21-23 April, 2010.

[14] L. Zhang, L. Yang, and T. Yang, "Cognitive Interference Management for LTE-A Femtocells with Distributed Carrier Selection," Proc. IEEE 72nd Vehicular Technology Conference Fall (VTC 2010-Fall), pp. 1-5, 6-9 Sept. 2010.

[15] S. Guruacharya, D. Niyato, E. Hossain, and D. I. Kim, "Hierarchical Competition in Femtocell-Based Cellular Networks," Proc. IEEE Globecom'10, Miami, FL, USA, 6-10 Dec. 2010.

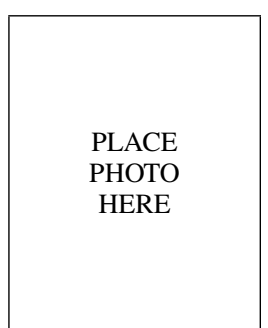

Nazmus Saquib received his B.Sc. degree in Electronics and Communication Engineering from BRAC University, Bangladesh, in 2008. He is currently working towards his M.Sc. degree in Electrical and Computer Engineering at the University of Manitoba, Winnipeg, MB, Canada. For academic excellence in undergraduate studies, Saquib won the Vice Chancellor's Gold Medal from BRAC University. Also, he has been awarded the University of Manitoba Graduate Fellowship. His research interests include interference management and resource allocation in OFDMA based heterogeneous networks.

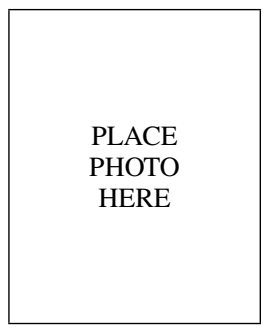

Ekram Hossain (S'98-M'01-SM'06) is a Professor in the Department of Electrical and Computer Engineering at University of Manitoba, Winnipeg, Canada. He received his Ph.D. in Electrical Engineering from University of Victoria, Canada, in 2001. Dr. Hossain's current research interests include radio resource management in wireless/mobile communications networks and cognitive radio systems (http://www.ee.umanitoba.ca/ ekram). He serves as the Area Editor for the IEEE Transactions on Wireless Communications in the area of "Resource Management and Multiple Access", an Editor for the IEEE Transactions on Mobile Computing, the IEEE Communications Surveys and Tutorials, and IEEE Wireless Communications. Dr. Hossain has several research awards to his credit which include the University of Manitoba Merit Award in 2010 (for Research and Scholarly Activities) and the 2011 IEEE Communications Society Fred Ellersick Prize Paper Award. He is a registered Professional Engineer in the province of Manitoba, Canada.

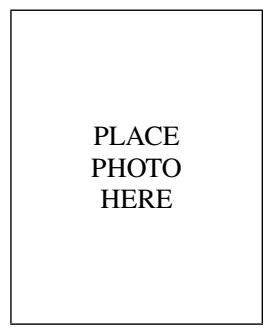

Long Bao Le (S'04-M'07) received the Ph.D. degree from University of Manitoba, Canada, in 2007. He is currently an Assistant Professor at INRS-EMT, University of Quebec, Montreal, Quebec, Canada. His current research interests include cognitive radio, cooperative diversity and relay networks, stochastic control and cross-layer design for communication networks. 


\begin{tabular}{|c|} 
\\
\\
PLACE \\
PHOTO \\
HERE \\
\end{tabular}

Dong In Kim (S'89-M'91-SM'02) is a Professor and SKKU Fellow in the School of Information and Communication Engineering at Sungkyunkwan University (SKKU), Suwon, Korea (http://wireless.skku.edu). He received his Ph.D. degree in Electrical Engineering from University of Southern California, Los Angeles, in 1990. From 2002 to 2007, he was a tenured Full Professor in the School of Engineering Science, Simon Fraser University, Burnaby, BC, Canada. His current research interests include cooperative communications, interference management for heterogeneous networks, cross-layer design and wireless security. He serves as an Editor for Spread Spectrum Transmission and Access for the IEEE Transactions on Communications and an Area Editor for Cross-layer Design and Optimization for the IEEE Transactions on Wireless Communications. He also serves as co-Editor-in-Chief for the Journal of Communications and Networks. 